\section{Spontaneous thrombosis of large splenorenal shunt during balloon-occluded retrograde transvenous obliteration in a patient with chronic persistent hepatic encephalopathy. Is this catheter assisted trans-venous occlusion?}

\author{
Lijesh Kumar, ${ }^{1}$ Cyriac Abby Philips, ${ }^{2}$ \\ Prakash Zacharias, ${ }^{3}$ Sudarshan Patil, ${ }^{3}$ \\ Philip Augustine ${ }^{2,3}$
}

${ }^{1}$ Department of Diagnostic and Interventional Radiology, ${ }^{2}$ Department of Hepatology and Transplant Medicine and ${ }^{3}$ Department of Gastroenterology, PVS Institute of Digestive Diseases, PVS Memorial Hospital, Kochi, Kerala, India

\begin{abstract}
Large spontaneous portosystemic shunts in cirrhosis are implicated in recurrent and/or chronic persistent hepatic encephalopathy. In long standing cases, these shunts lead to portal vein thrombosis and hepatic dysfunction. Balloon-occluded retrograde transvenous obliteration (BRTO) is an endovascular technique that is usually employed for shunt closure in the patients manifesting the features of chronic hepatic encephalopathy. There are several reports documenting systemic and portal vein thrombosis as a part of the procedure. We report first time a patient in whom the difficult and partial BRTO procedure led to the extensive thrombosis of the large splenorenal shunt itself without sclerosant instillation.
\end{abstract}

\section{Introduction}

Hepatic encephalopathy is a syndrome of complex neuropsychiatric manifestations associated with hepatic dysfunction or large porto-systemic shunts (PSS, congenital, spontaneous, or iatrogenic). ${ }^{1}$ Large spontaneous PSS in cirrhotics lead to a syndrome initially christened by Kumamoto and coworkers as Porto-Systemic Shunt Syndrome (PSSS). The early stages are marked by recurrent episodes of encephalopathy in the absence of liver failure; while late and very late stages are characterized by more frequent episodes of encephalopathy or chronic persistent encephalopathy in the presence of portal vein thrombosis and hepatic dys- function (Saad Classification). ${ }^{2}$ Large PSS can be associated with esophageal, gastric or ectopic varices, and those that do not pass through the walls of viscera are not associated with ectopic varices. The most common type of shunt is the gastro-renal shunt (GRS) with isolated gastric or gastroesophageal varices. Spontaneous splenorenal shunts (SSRS) are also associated with gastro-esophageal or ectopic varices. Portosystemic shunts have two important clinical manifestations in the patients of portal hypertension and cirrhosis. They either contribute to a difficult to manage acute variceal bleeding or results in recurrent and chronic hepatic encephalopathy. In either case, the balloon occluded transvenous obliteration of these shunts with variceal embolization remains an important aspect in management. ${ }^{3}$ Shunt occlusion is traditionally done by BRTO, wherein GRS is accessed through the left renal vein from a transjugular or transfemoral route and balloon catheter occlusion followed by sclerosant injection for obliteration is performed. There are certain modifications of BRTO, which are employed in the occlusion of other types of spontaneous shunts, namely balloon-occluded anterograde transvenous occlusion (BATO, through direct percutaneous portal vein approach), coil assisted (CARTO) or plug assisted (PARTO) transvenous obliteration. In the presence of a patent portal vein and varices, PSS should be obliterated with BRTO technique. The bland occlusion of a shunt with coils or vascular plugs could lead to a high variceal pressure and catastrophic bleeding. However, in the absence of varices, CARTO or PARTO are the safest options. ${ }^{4}$ The complications of BRTO include variceal bleeding, ascites, and the worsening of liver function. Local complications include the thrombosis of systemic or portal veins. Herein, we present an interesting patient with chronic persistent hepatic encephalopathy associated with large SSRS in whom an incomplete or difficult BRTO procedure was carried out without balloon occlusion or sclerosant instillation, which led to spontaneous thrombosis of the whole shunt with partial thrombosis of splenic vein. The former aspect has not yet been described in the literature [catheter associated transvenous occlusion (CATO)].

\section{Case Report}

A 52-year-old man diagnosed with cirrhosis secondary to alcohol use having a history of recurrent attacks of alteration in sensorium was hospitalized thrice in the preceding three weeks. One month ago, his
Correspondence: Cyriac Abby Philips, Department of Hepatology and Transplant Medicine, PVS Institute of Digestive Diseases, PVS Memorial Hospital, Kochi 682017, Kerala, India.

E-mail: abbyphilips@gmail.com

Key words: cirrhosis, portal hypertension, portosystemic shunts, hepatic encephalopathy, BRTO, shunt occlusion

Contributions: the authors contributed equally.

Conflict of interest: the authors declare no potential conflict of interest.

Received for publication: 26 September 2016 Accepted for publication: 14 December 2016.

This work is licensed under a Creative Commons Attribution NonCommercial 4.0 License (CC BY-NC 4.0).

(C) Copyright L. Kumar et al., 2017

Licensee PAGEPress, Italy

Gastroenterology Insights 2017; 8:6906 doi:10.4081/gi.2017.6906

wife noticed recurrent slurring of speech, symmetric tremors of both upper and lower limbs, lack of movements and slow responses to commands. Three months ago, the patient complained memory loss and sleep reversal to a local physician. He quitted alcohol use four months ago and was not a substance abuser. On admission to our hospital, clinical examination revealed him as a disoriented patient with slurred speech, masked facies, upper and lower limb rigidity without cranial nerve deficits or focal motor weakness and a modified Rankin score (mRS) of 5. His blood chemistry tests revealed arterial ammonia $(138 \mu \mathrm{g} / \mathrm{dL}$; normal $<75 \mu \mathrm{g} / \mathrm{dL})$, aspartate aminotransferase (AST, $52 \mathrm{U} / \mathrm{L}$; normal 7-38 U/L) and alanine aminotransferase (ALT, $39 \mathrm{U} / \mathrm{L}$; normal 4-43 U/L). The blood counts, blood cultures, urinalysis, serum sodium, potassium, magnesium, ionized calcium concentrations and blood gas analyses were normal. An abdominal ultrasound showed cirrhotic liver with splenomegaly and peri-splenic collaterals without ascites. The computed tomography of the brain showed diffuse mild cerebral atrophy, while a bedside electroencephalogram did not show any epileptiform activity. The magnetic resonance imaging of the brain was suggestive of the symmetric $\mathrm{T} 1$ hyperintensities of the basal ganglia, caudate and putamen, consistent with chronic hepatic encephalopathy. The patient was managed medically with oral disaccharides, lactulose enemas, and intravenous L-ornithine-L-arginine with added 
zinc and thiamine supplementation without improvement in rigidity, tremors or sensorium after 72 hours. Subsequently, the computed tomography of the abdomen was performed, which revealed a large tortuous splenorenal shunt $(1.4 \mathrm{~cm})$, multiple perisplenic collaterals, hugely dilated left renal vein in the background of cirrhosis and portal hypertension (Figure 1A-C) without any evidence of hepatocellular carcinoma or portal vein thrombosis. Upper gastrointestinal endoscopy revealed grade 1 esophageal varices but no gastric or ectopic varices. In the view of PSSS and hepatic Parkinsonism, the patient was planned to undergo BRTO. A written informed consent was obtained from the patient. Under conscious sedation, right common femoral vein was accessed and a $6 \mathrm{~F}$ vascular access sheath (Cook Medical, Bloomington, Indiana) was placed. The selective cannulation of the left renal vein was performed with the use of a $5 \mathrm{~F}$ angiographic catheter $(\mathrm{C} 2$ Cook Medical) which was then replaced with a flexible straight sheath (12 F, $45 \mathrm{~cm}$, Flexor Check-Flo, Mullin design, Cook Medical). Thereafter, a 4F angled glide catheter (MPA/C2 Slip Cath, Cook Medical) was employed for shunt cannulation (Figure 1D). The acute angulation of the collateral opening into the shunt made the cannulation difficult. A $4 \mathrm{~F}$ curved glide catheter was then used to correctly cannulate the shunt. The glide catheter was then replaced with a balloon catheter over a stiff guide wire (AES, Cook Medical). However, the introduction of the balloon catheter was found to be difficult due to the posteriorly placed tortuous splenic collateral complex acutely opening into the shunt. The exchange of the flexible straight sheath with Ansel modification of the Mullin design (Cook Medical) was also futile in balloon catheter introduction. We also used Equalizer balloon ${ }^{\circledR}$ (Boston Scientific, Massachusetts, USA) and Reliant ${ }^{\circledR}$ balloon $(10 \mathrm{~mm}$, Medtronic, Minnesota, USA) in addition to biliary extraction balloon (Boston Scientific, Massachusetts, USA). In order to avoid a prolonged requirement of anesthesia, the procedure was abandoned and another attempt was made after 48 hours. This time again the repeat attempt of cannulating the shunt was found to be futile. An intra-procedural Doppler ultrasonography showed partial thrombosis of the splenic vein at the junction of the splenorenal shunt with the complete thrombosis of the collateral complex and the splenorenal shunt (Figure 1E). The computed tomography of the abdomen confirmed these findings (Figure 1F). One week after the procedure, the shunt remained completely thrombosed with improvement in the grade of hepatic encephalopathy from 3 to 1, arterial ammonia decreased to $98 \mu \mathrm{g} / \mathrm{dL}$ with improvement in $\mathrm{mRS}$ to 3 .

\section{Discussion}

The closure of large SPSS with BRTO technique has been found to improve recurrent and chronic persistent hepatic encephalopathy. The procedure leads to thrombotic occlusion of the varices when the sclerosant traverses through the PSS while the variceal blood flow is halted in the presence of retrograde balloon occlusion. The feeders of the variceal complex also thrombose in the presence of adequate stagnation and reflux of sclerosant within the shunt. The veins of the systemic and portal system in a close proximity of the shunt complex are also affected by thrombus extension. ${ }^{5}$ This aspect of BRTO was studied by Cho et al. In their study on 60 patients with liver cirrhosis undergoing BRTO to treat gastric variceal bleeding, 14 thrombi developed in 13 systemic and portal veins of nine patients $(15 \%)$ post procedure during the follow up period. The affected regions included splenic vein, left renal vein, and main portal vein. The authors also described the thrombus based on $\mathrm{CT}$ attenuation values. During the follow up (range 5-25 months), highly attenuated thrombi (possibly iodized oil deposit) disappeared completely, while low attenuated thrombi (true thrombus) partially resolved or progressed completely to occlude the affected vein. ${ }^{6}$

However, in our patient, we could not complete the BRTO procedure due to the difficult collateral anatomy during the first attempt and due to the complete obliteration of the shunt system during the second attempt. Our patient underwent prolonged and multiple catheter manipulations within the shunt and the variceal systems as the part of wire exchanges. Vascular injury secondary to the catheter and wire manipulations leading to thrombosis within splenoportal venous system (but not in large shunts) has been reported earlier in BRTO patients. ${ }^{7}$ In our patient, it was surprising to find that the inadvertent catheter related obliteration of the venous shunt system had already taken place during the second attempt - a goal we aimed to achieve with the BRTO procedure - making the current procedure a sort of Catheter Assisted Transvenous Obliteration (CATO) of the shunt. In our patient, such total obliteration was possible mainly as the shunt was not associated with esophageal, gastric or ectopic varices, thereby allowing for extensive thrombus development within the

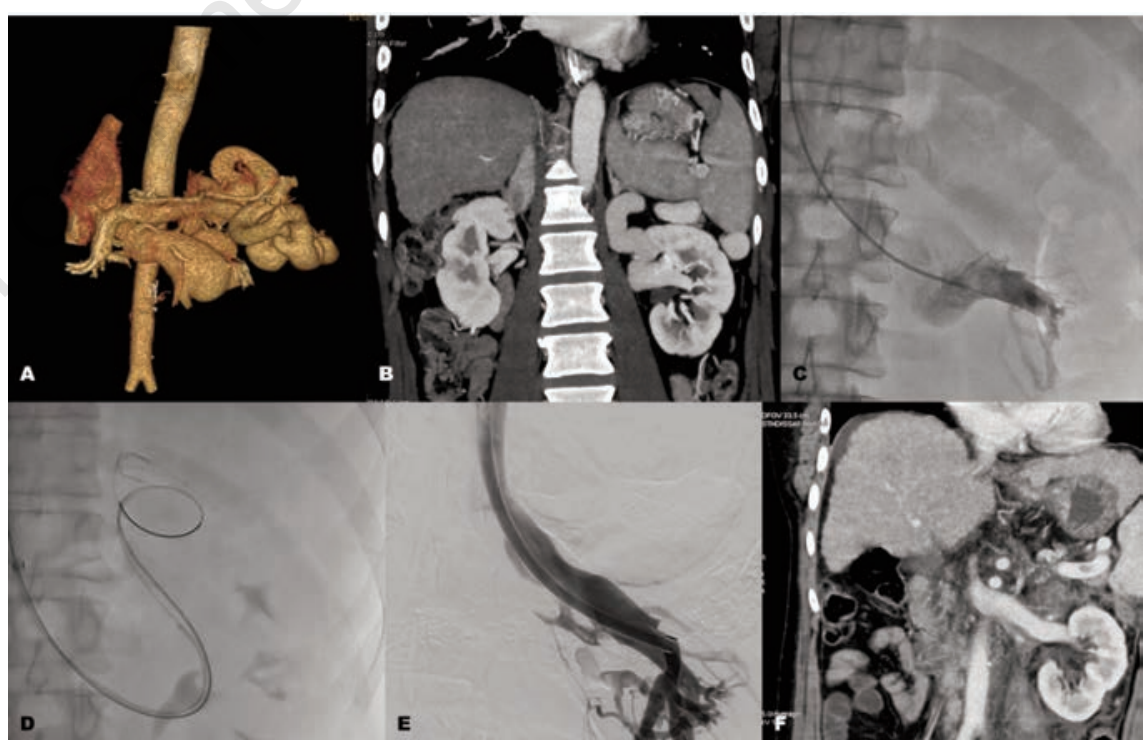

Figure 1. Computed tomography (CT) three-dimensional reconstruction of the collateral system and spleno-renal shunt (A); large tortuous spleno-renal shunt arising from the left renal with dilated left renal vein of $C$ T venography $(B)$; fluoroscopic real time image of dilated left renal vein with contrast dye escape into the shunt without opacification of inferior vena cava (IVC, C); multiple guide wire introduction into the large shunt through cannulation of tortuous collateral (D); fluoroscopic real time image of shunt cannulation 48 hours later, after the first attempt, revealing complete collapse of the collaterals and shunt with opacification of less dilated left renal vein and IVC (E); corresponding CT venography revealing complete shunt thrombosis and collateral system collapse without renal vein dilatation (in comparison to $B$ ). 
whole shunt system.

To the best of our knowledge, this is the first report on catheter associated total obliteration of large SPSS in a cirrhotic patient. However, how long such obliteration lasts without recanalization remains to be seen during the follow up. In such a scenario, we advise to perform CARTO, which is quicker and with minimal adverse events. This accidental shunt obliteration however simulated a functional BRTO, leading to the improvement in hepatic encephalopathy grades and disability scores in the patient. In future, in difficult to perform BRTO cases, CATO could be a temporizing salvage technique.

\section{References}

1. Saad WEA. The history and evolution of balloon-occluded retrograde transve- nous obliteration (BRTO): from the United States to Japan and Back. Semin Interv Radiol 2011;28:283-7.

2. Saad WE. portosystemic shunt syndrome and endovascular management of hepatic encephalopathy. Semin Interv Radiol 2014;31:262-5.

3. Al-Osaimi AMS, Sabri SS, Caldwell $\mathrm{SH}$. Balloon-occluded retrograde transvenous obliteration (BRTO): preprocedural evaluation and imaging. Semin Interv Radiol 2011;28:288-95.

4. Saad WEA, Sze DY. Variations of balloon-occluded retrograde transvenous obliteration (BRTO): balloon-occluded antegrade transvenous obliteration (BATO) and alternative/adjunctive routes for BRTO. Semin Interv Radiol 2011;28:314-24.

5. Cho SK, Shin SW, Do YS, et al. Development of thrombus in the major systemic and portal veins after balloonoccluded retrograde transvenous obliteration for treating gastric variceal bleeding: its frequency and outcome evaluation with CT. J Vasc Interv Radiol 2008;19:529-38.

6. Laleman W, Simon-Talero M, Maleux $\mathrm{G}$, et al. EASL-CLIF-Consortium. Embolization of large spontaneous portosystemic shunts for refractory hepatic encephalopathy: a multicenter survey on safety and efficacy. Hepatology 2013;57:2448-57.

7. Mukund A, Rajesh S, Arora A, et al. Efficacy of balloon-occluded retrograde transvenous obliteration of large spontaneous lienorenal shunt in patients with severe recurrent hepatic encephalopathy with foam sclerotherapy: initial experience. $\mathrm{J}$ Vasc Interv Radiol 2012;23:1200-6. 